\title{
In Vitro Photodynamic Therapy with Chlorin e6 Leads to Apoptosis of Human Vascular Smooth Muscle Cells
}

\author{
Magdalena Wawrzyńska • Wojciech Kałas • \\ Dariusz Biały · Ewa Zioło • Jacek Arkowski • \\ Walentyna Mazurek • Leon Strządała
}

Received: 30 October 2008/Accepted: 15 July 2009/Published online: 14 January 2010

(C) L. Hirszfeld Institute of Immunology and Experimental Therapy, Wroclaw, Poland 2010

\begin{abstract}
Percutaneous coronary intervention has become the most common and widely implemented method of heart revascularization. However, the development of restenosis remains the major limitation of this method. Photodynamic therapy (PDT) recently emerged as a new and promising method for the prevention of arterial restenosis. Here the efficacy of chlorin e6 in PDT was investigated in vitro using human vascular smooth muscle cells (TG/HA-VSMCs) as one of the cell types crucial in the development of restenosis. PDT-induced cell death was studied on many levels, including annexin $\mathrm{V}$ staining, measurement of the generation reactive oxygen species (ROS) and caspase-3 activity, and assessment of changes in mitochondrial membrane potential and fragmentation of DNA. Photosensitization of TG/HA-VSMCs with a $170 \mu \mathrm{M}$ of chlorin e6 and subsequent illumination with the light of a 672-nm diode laser $\left(2 \mathrm{~J} / \mathrm{cm}^{2}\right)$ resulted in the generation of ROS, a decrease in cell membrane polarization, caspase- 3 activation, as well as DNA fragmentation. Interestingly, the latter two apoptotic events could not be observed in photosensitized and illuminated NIH3T3 fibroblasts, suggesting different outcomes of the model of PDT in various types of cells. The results obtained with human VSMCs show that chlorin e6 may be
\end{abstract}

M. Wawrzyńska and W. Kałas contributed equally to this work.

M. Wawrzyńska · D. Biały · J. Arkowski · W. Mazurek Department of Cardiology, Wrocław Medical University, Wroclaw, Poland

W. Kałas $(\bowtie) \cdot$ E. Zioło · L. Strządała

Ludwik Hirszfeld Institute of Immunology and Experimental

Therapy, Polish Academy of Sciences, Weigla 12,

53-114 Wroclaw, Poland

e-mail: kalas@iitd.pan.wroc.pl useful in the PDT of aerial restenosis, but its efficacy still needs to be established in an animal model.

Keywords Restenosis - Photodynamic therapy ·

Cell death · Chlorin · Vascular smooth muscle cells

\author{
Abbreviations \\ PDT Photodynamic therapy \\ PCI Percutaneous coronary intervention \\ PI Propidium iodide \\ ROS Reactive oxygen species \\ VSMCs Vascular smooth muscle cells
}

\section{Introduction}

Ischemic heart disease caused by coronary artery atherosclerosis and its complications is the leading cause of morbidity and mortality in developed countries. During the last two decades, percutaneous coronary intervention (PCI) has become the most common and widely implemented method of heart revascularization (Hill et al. 2007). Restenosis, which occurs in the initial 3-24 months after revascularization in about $30-50 \%$ of cases, remains the major limitation of the method (Moussavian et al. 2001).

Although the molecular basis of restenosis is not yet fully understood, it is known that it is an inflammatory and multifactoral process involving the activation and proliferation of intimal smooth muscle cells and the migration of adventitial cells (fibroblasts) into the subintimal space, which results in the production and deposition of extracellular matrix elements and thus neointimal hyperplasia, eventually leading to late lumen loss (Schiele 2005; Toutouzas et al. 2004; Zubilewicz et al. 2001). Because 
intimal hyperplasia consists mainly of proliferating vascular smooth muscle cells (VSMCs), control of their proliferation or their elimination may be a good target in the therapy of restenosis. It is worth mentioning that over 50 agents were shown to modulate intimal hyperplasia and restenosis in animal models favorably, but few have been shown to be effective in humans (Sobeh et al. 1995).

Photodynamic therapy (PDT) is an alternative and novel approach to modulate the vascular response to injury caused by PCI (Mansfield et al. 2001; Moussavian et al. 2001) and has already been clinically approved for the treatment of various proliferative diseases in oncology, ophthalmology, and dermatology. PDT involves a photosensitizing agent that accumulates selectively in the target cells and its illumination with a low-power laser of appropriate wavelength (preferably close to red) to generate cytotoxic reactive oxygen species (ROS). The effect of PDT depends on the photosensitizing agent, its concentration, as well as the cell type. PDT has been shown to reduce proliferation and to induce cell-cycle arrest, apoptosis, autophagy, as well as necrosis-like cell death (Buytaert et al. 2007; Granville et al. 2001a; Granville et al. 2001b; Heckenkamp et al. 1999; Kessel and Reiners 2007; LaMuraglia et al. 2000). Several types of photosensitizer, such as porphycene derivatives, 5-aminolevulinic acid, photophrin II, chloroaluminium sulfonated phtalocyanine, and $\mathrm{Zn}$ (II)-phtalocyanine, have been tested and proved to be effective for photonangioplasty in animal models (Chen et al. 2001; Derkacz et al. 2005; Eton et al. 1996; Kereiakes et al. 2003; Magaraggia et al. 2006; Nagae et al. 2001). Chlorin e6 (Fig. 1) is a member of the chlorin family with a characteristic Soret band absorption at $400 \mathrm{~nm}$ and less intense Q bands in the visible region of $450-700 \mathrm{~nm}$ (Cunderlíková et al. 1999). Illumination of chlorin e6 results in the generation of an exited singlet-state molecule, which results in the formation of highly reactive singlet oxygen and the formation of ROS. The resulting oxidative stress leads to impairment of mitochondria and endoplasmic reticulum function and, consequently, to execution of the cell-death program (Buytaert et al. 2007; Gurinovich et al. 1992; Kessel and Poretz 2000; Kessel et al. 1995).

From the few clinical experimental data on the influence of PDT on vascular cells it is postulated that it induces apoptotic processes in cells and thus results in loss of cell viability (Granville et al. 2001a; Kessel et al. 2000). This observation potentially favors PDT over other techniques, as apoptosis does not induce much inflammatory response within the vessel wall, which enables maintaining vascular wall integrity. Chlorin e6 has good water and lipid solubility properties and easily translocates through the cell membrane. In cells, chlorin e6 has been shown to localize to the membrane (Cunderlíková et al. 1999) and mitochondria (Kessel and Poretz 2000). In a few experiments with animal models, PDT with chlorin e6 was demonstrated to inhibit arterial intimal hyperplasia after both balloon injury and stent implantation (Heckenkamp et al. 2004; Nagae et al. 2001; Wakamatsu et al. 2005), but no data are available on the molecular effects of the action of chlorin e6 on human vascular wall cells.

This paper presents a molecular in vitro study aimed at assessing the time- and dose-dependent influence of PDT with chlorin e6 on fibroblasts and human smooth muscle cells. We show a rapid generation of ROS after the illumination of photosensitized cells and subsequent events, including cellular membrane depolarization, mitochondrial potential loss, caspase-3 activation, and DNA fragmentation. When PDT conditions optimal for vascular cells were applied to PDT of NIH3T3 fibroblasts, we observed the induction of toxicity and phosphatidylserine presentation, but not other apoptotic hallmarks such as DNA fragmentation or caspase-3 activation. The present paper demonstrates that TG/HA-VSMCs undergo apoptosis as a consequence of PDT, indicating the value of chlorin e6 for animal studies but also showing possible drawbacks due to differences in response of various cell populations to the PDT.

\section{Materials and Methods}

\section{Cell Cultures and Reagents}

T/G HA-VSMCs, a human aortic smooth muscle cell line established by telomerase transfection, were cultured in F12 K medium supplemented with $2 \mathrm{mM}$ L-glutamate (Gibco, Invitrogen Corp., USA) and $0.1 \mathrm{mg} / \mathrm{ml}$ heparin (Sigma-Aldrich, USA), $0.03 \mathrm{mg} / \mathrm{ml}$ endothelial cell growth supplement (Sigma-Aldrich, USA), and 10\% fetal calf serum (FCS; Gibco, USA). NIH3T3 mouse embryonal fibroblasts were cultured in a 10-ml flask in Dulbecco's Modified Eagle's Medium (Ludwik Hirszfeld Institute of Immunology and Experimental Therapy, Poland) supplemented with 10\% FCS (Gibco, USA), 3\% glutamate, $10 \mathrm{mM}$ HEPES, $1 \mathrm{mM}$ sodium pyruvate (all SigmaAldrich, USA). The photosensitizer chlorin e6 (fotolon, 18-carboxy-20-(carboxymethyl)-8-ethenyl-13-ethyl-2,3,dihydro- 3,7,12,17-tetramethyl-21H, 23H-porphin-2-propionic acid; Haemato Polska) was prepared fresh in $0.9 \%$ $\mathrm{NaCl}$ (POCh, Poland). Also used were 96- and 24-well plates, $10 \mathrm{ml}$ flasks (Corning Costar, The Netherlands), phosphate-buffered saline solution (PBS; Ludwik Hirszfeld Institute of Immunology and Experimental Therapy, Poland), Annexin V-FITC conjugate, and propidium iodide (PI; Sigma-Aldrich, USA). 
Fig. 1 a Structure of chlorin e6. The presence of the chlorin ring gives the compound fluorescent and photochemical properties. b Chlorin e6 distribution in the cell may be directly observed using a fluorescence microscope equipped with a UV lamp and 605-670 nm filter. Chlorin e6 rapidly translocates through the cell membrane into the cell and accumulates in the cytoplasm. Representative microphotography is shown

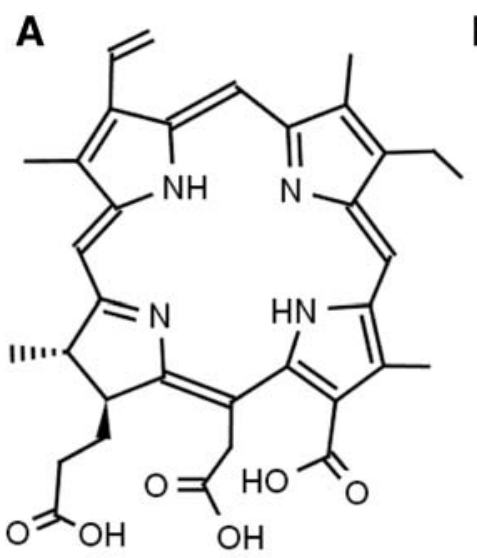

B

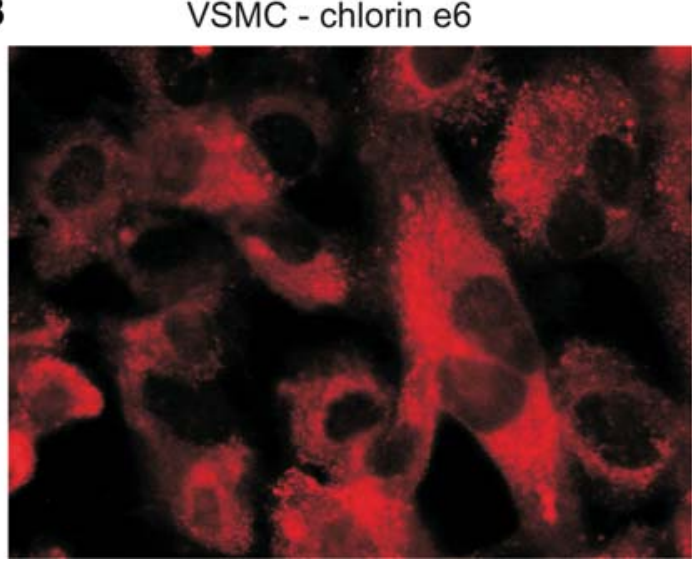

\section{In Vitro PDT Experiments}

In the dark and using coated tubes, plates, etc., 200,000 cells (in a 24-well plate) were pretreated with the indicated concentration of chlorin e6 for $90 \mathrm{~min}$ in culture medium. Then the plate was washed twice with warm PBS containing $2.5 \%$ FCS and incubated for $30 \mathrm{~min}$. The cells were illuminated in a darkened room with 672-nm diode laser light at a fluence rate of $75 \mathrm{~mW} / \mathrm{cm}^{2}$ or $150 \mathrm{~mW} / \mathrm{cm}^{2}$ to achieve total light doses of $0.6,1.1$, and $2 \mathrm{~J} / \mathrm{cm}^{2}$.

\section{Microscopy}

To prove the presence and assess the distribution of chlorin e6 in cells, the cells $(10,000 /$ well, $250 \mu \mathrm{l})$ were cultured on eight-chamber microscope slides (Nunc, USA). After 1 day of culture the cells were treated with $17 \mu \mathrm{M}$ of chlorin e6 for $10 \mathrm{~min}$ and washed twice with warm PBS. The presence of chlorin e6 inside the cell was assessed using a Carl Zeiss fluorescence microscope equipped with a UV lamp and 43HS Filter Set (Carl Zeiss MicroImaging Inc., Germany).

\section{MTS Assay}

The toxicity of the PDT was assayed by the MTS assay (Promega, USA) according to the supplier's instructions. Briefly, 5,000 cells/well were cultured and subjected to the PDT experiment. After $35 \mathrm{~min}$ of incubation, $20 \mu \mathrm{l}$ of MTS reagent was added. After 2 h, 490-nm absorbance was read on a Vallac Victor2 plate reader.

\section{Early Apoptosis Assessment by Annexin V Staining}

Cells subjected to PDT were cultured for $45 \mathrm{~min}$ and then detached using $1 \%$ trypsin (Gibco), washed twice with PBS containing 2.5\% FCS, and stained with $50 \mu \mathrm{g} / \mathrm{ml}$ Annexin $\mathrm{V}$-FITC conjugate in the presence $10 \mu \mathrm{g} / \mathrm{ml}$ PI for $10 \mathrm{~min}$ at room temperature. The stained cells were analyzed using a FACSCalibur flow cytometer (Becton-Dickinson, USA) and WinMDI 2.8 software.

\section{Caspase-3 Activity}

Caspase-3 activity in cell lysates was measured using a Caspase-3 Cellular Activity Kit (Calbiochem, USA) according to the manufacturer's instructions. After illumination, the cells were incubated for $4 \mathrm{~h}$ and then lysed. Caspase-3 Inhibitor I (Ac-DEVD-CHO) was used to test the specificity of the caspase activation. The samples were analyzed on a Vallac Victor2 plate reader. The readings were converted to caspase-3 activity units (U) in $100 \mu \mathrm{g}$ of protein according to a standard curve prepared using the supplied human recombinant caspase-3.

\section{Measurement of ROS}

ROS concentration was assessed by measuring the 485-/ 535-nm fluorescence of $\mathrm{H}_{2}$ DCFDA [6-carboxy-2 $2^{\prime}, 7^{\prime}$ dichlorodihydrofluorescein diacetate, di(acetoxymethyl ester); Molecular Probes, USA]. Briefly, 5,000 cells (96-well plate) were incubated in the presence of $170 \mu \mathrm{M}$ chlorin e6 and $10 \mu \mathrm{M}$ of $\mathrm{H}_{2}$ DCFDA for $30 \mathrm{~min}$ in PBS with $1 \%$ FCS. Then the cells were washed twice with warm PBS with $2.5 \%$ FCS, cultured for 30 min in medium and illuminated. Fluorescence readings were performed directly after illumination. A blank-probe (cells and medium) reading was used as the background and subtracted from all the sample readings.

\section{Measurement of Mitochondrial Membrane Potential}

After washing with PBS, JC-1 $\left(5,5^{\prime}, 6,6^{\prime}\right.$-tetrachloro1,1',3,3'-tetraethyl-benzimidazolylcarbocyanine iodide, $1 \mu \mathrm{M}$; Molecular Probes, USA) was added to cells subjected to PDT and the cells were incubated for $15 \mathrm{~min}$ at $37^{\circ} \mathrm{C}$. 
The cationic dye JC-1 exhibits potential-dependent accumulation in the mitochondria. It indicates mitochondria depolarization by a decrease in the red to green fluorescence intensity ratio. The cell suspensions were analyzed with a FACSCalibur flow cytometer (Becton-Dickinson, USA) according to the manufacturer's instructions.

\section{Detection of DNA Fragmentation}

The detection of apoptosis was based on evaluating DNA content with the use of PI and flow cytometry. Briefly, after $18 \mathrm{~h}$ of treatment, the cells were washed twice with PBS containing $2.5 \%$ FCS, fixed with $70 \%$ ethanol (POCh, Poland) for $30 \mathrm{~min}$ at $4{ }^{\circ} \mathrm{C}$, washed again with PBS, and then stained with $10 \mathrm{ug} / \mathrm{ml}$ PI (50 without $\mathrm{Ca}^{2+}, \mathrm{Mg}^{2+}$; Sigma-Aldrich, USA) overnight at $4^{\circ} \mathrm{C}$. The cell suspensions were analyzed with a FACSCalibur flow cytometer (Becton-Dickinson, USA). DNA content was evaluated on the basis of FL-2 histograms using WinMDI 2.8 software.

\section{Results}

Chlorin e6 is Detectable Inside Cells As Early As 15 min After Treatment

The efficiency of the PDT model was studied using a human aortic smooth muscle cell line (T/G HA-VSMC), representing one of the cell types playing a crucial role in the development of restenosis (Schiele 2005). Chlorin e6, as a member the chlorin group, has strong photochemical and fluorescence properties (Cunderlíková et al. 1999) (Fig. 1a). Thus, using a fluorescence microscope we were able to observe the cellular localization of chlorin e6. We found that even $10 \mathrm{~min}$ of incubation of TG/HA-VSMCs with $17 \mu \mathrm{M}$ chlorin e6 is sufficient for its cellular uptake (Fig. 1b). The pattern of fluorescence indicates a cytoplasmic localization of the compound, probably bound to membrane structures. It is worth noting that a further increase in incubation time had no effect on the observed fluorescence intensity or staining distribution (data not shown).

PDT with Chlorin e6 Leads to the Generation of ROS in Photosensitized Cells

We designed a model of PDT consisting of photosensitizing, washing, incubation, and illumination. The scheme of the PDT experiment is shown in Fig. 2a. In an aqueous environment, illumination of chlorin e6 leads to the generation of ROS, which is the basis of the PDT (Kessel and Poretz 2000). We monitored ROS produced due to the illumination of photosensitized TG/HA-VSMCs and found

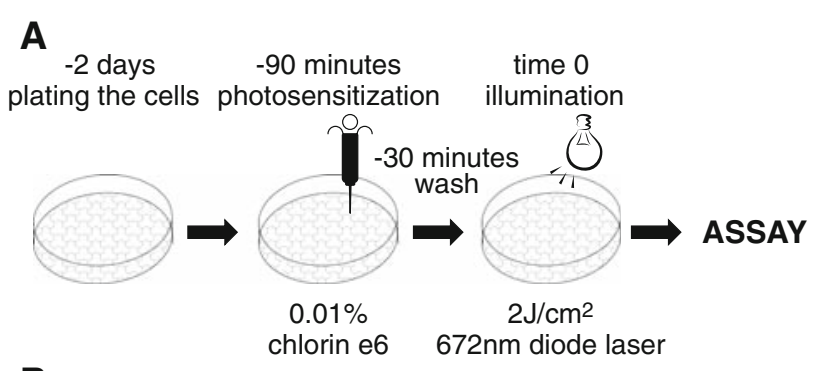

B

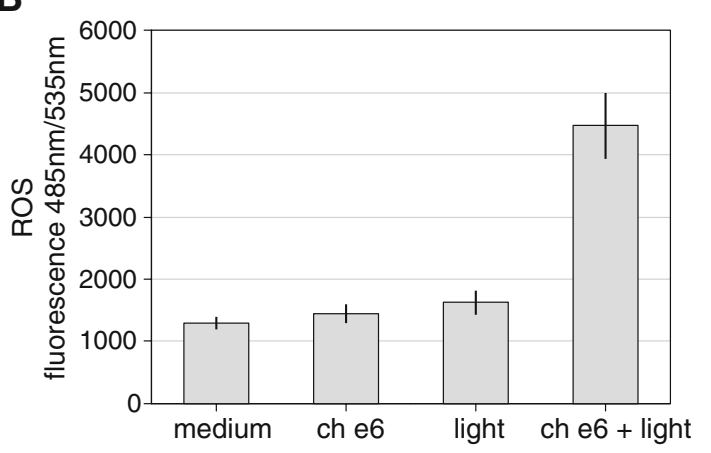

Fig. 2 a Scheme of the standard in vitro photodynamic therapy experiment. Cells were plated 2 days prior to photosensitization, then incubated for $1 \mathrm{~h}$ in the presence of chlorin e6, washed, incubated for $30 \mathrm{~min}$, and illuminated in a dark room. b The generation of reactive oxygen species (ROS) due to illumination of the photosensitized TG/HA-VSMCs. The dichlorofluorescein derivate, carboxy$\mathrm{H}_{2}$ DCFDA was used as the ROS detection reagent. A fourfold increased in 485-/535-nm fluorescence right after illumination of TG/HA-VSMCs was detected. ch e6 $170 \mu \mathrm{M}$ chlorin e6, light 672-nm diode laser, $2 \mathrm{~J} / \mathrm{cm}^{2}$

a rapid threefold increase in fluorescence due to the generation of free radicals immediately after the illumination of the smooth muscle cells (Fig. 2b). In non-illuminated cells we observed no increase in ROS concentration.

\section{Phototoxicity of PDT with Chlorin e6} in Human TG/HA-VSMCs

Various combinations of chlorin e6 concentration, ranging from 17 to $170 \mu \mathrm{M}$, and light doses of $0.6,1.1$, and $2 \mathrm{~J} / \mathrm{cm}^{2}$ were examined. The toxicity of PDT was determined by measuring dehydrogenase enzyme activity using the colorimetric MTS assay. We found that chlorin e6 alone does not induce significant toxicity (Fig. 3a). Illumination alone also did not induce toxicity measured by the MTS assay (Fig. 3b). The TG/HA-VSMC model of PDT showed almost linear relationships between light dose or chlorin e6 concentration and toxicity. Increasing the concentration of chlorin e6 resulted in a gradual increase in toxicity when the same light dose $\left(2 \mathrm{~J} / \mathrm{cm}^{2}\right)$ was used (Fig. 3a). Similarly, in cells photosensitized with $170 \mu \mathrm{M}$ chlorin e6, increasing the light dose led to a gradual increase in toxicity (Fig. 3b). The maximum effect of about $80 \%$ toxicity was observed for $170 \mu \mathrm{M}$ chlorin e6 and a light dose of $2 \mathrm{~J} / \mathrm{cm}^{2}$, and 
A

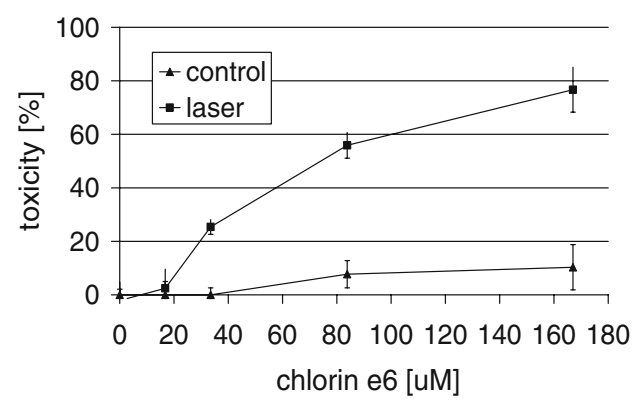

Fig. 3 Testing and optimization of parameters for the in vitro photodynamic therapy of TG/HA-VSMC determined by MTS assay. Toxicity of PDT induced in TG/HA-VSMC culture (MTS assay) using: a $17-170 \mu \mathrm{M}$ concentrations of chlorin e6 (squares) and in

these conditions were used in the subsequent experiments. These data suggest that there is no need to increase the drug dose for the PDT above $170 \mu \mathrm{M}$, as it may result in dark toxicity.

\section{Cells Subjected to PDT Had Properties of Apoptotic Cells}

Both necrotic and apoptotic processes may be initiated by the ROS generated by the illumination of photosensitized cells. To discover the nature of the observed toxicity, we studied a few aspects of apoptosis: phosphatidylserine presentation, mitochondrial potential impairment, caspase3 activation, and DNA fragmentation (Fig. 4). Staining with Annexin V and PI was performed to detect cells at an early stage of apoptosis. We found that the illumination of photosensitized cells leads to an increase in the population of cells expressing phosphatidylserine (59\% vs ca. $10 \%$ in controls); at the same time there was also a slight increase in both Annexin V-PI double-positive and PI-positive cells, indicating a decrease in cell membrane integrity (Fig. 4a). Further incubation (e.g. 4 h, data not shown) shifts the Annexin V-positive population to Annexin V-PI doublepositive, showing a gradual loss of cell membrane integrity during that time.

Programmed cell death is very often initiated by or related to loss of function by the mitochondria, which is reflected by a decrease in mitochondrial membrane potential. It is known that ROS accumulation may be a cause of disruption of mitochondrial function. This can be studied using IC-1 dye, which changes color according to the state of mitochondrial potential. We observed significant impairment of mitochondrial potential in about $50 \%$ of the cells $3 \mathrm{~h}$ after PDT (Fig. 4b). In contrast to Annexin V/PI staining, in which treatment with photosensitizer or light alone did not lead to any changes in staining pattern,
B

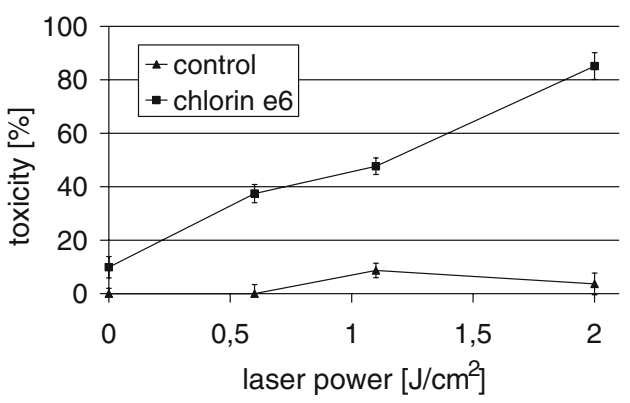

combination with a light dose of $2 \mathrm{~J} / \mathrm{cm}^{2}$ applied using a 672-nm diode laser (triangles) or $\mathbf{b}$ testing the light doses of 0.6, 1.1, and $2 \mathrm{~J} / \mathrm{cm}^{2}$ in the presence (triangles) or absence (squares) of $170 \mu \mathrm{M}$ chlorin e6

we could observe here a slight but recurrent increase in the population of cells with impaired mitochondrial potential in the photosensitized but non-illuminated samples.

It is known that the disruption of mitochondria leads to cytochrome $c$ release to the cytoplasm, which activates a cascade of caspases with the effector caspase- 3 at the bottom. We examined the activation of effector caspase-3 in the TG/HA-VSMCs subjected to PDT and found an approximately threefold activation of caspase- 3 in the photosensitized and illuminated cells, but not in the illuminated control cells (Fig. 4C). Here we could also observe a slight but recurrent increase in caspase activity in photosensitized cells, indicating that chlorin e6 alone may induce an apoptotic response.

The most known hallmark of apoptosis is DNA fragmentation. We found that our model of PDT leads to DNA fragmentation in about $70 \%$ of cells $18 \mathrm{~h}$ after illumination (Fig. 4d), indicating that chlorin e6-based PDT results in classical apoptosis of VSMCs. Light or chlorin e6 alone had no impact on DNA status.

\section{Chlorin e6 PDT Results in Atypical Programmed Cell Death in NIH3T3 Fibroblasts}

We decided to test our previous observations on other cells. We choose fibroblasts as they may represent another cell population present in restenotic plaque. We examined the influence of chlorin e6 PDT $(170 \mu \mathrm{M}$ of chlorin e6 in combination with a $2-\mathrm{J} / \mathrm{cm}^{2}$ light dose) on NIH3T3 cells, widely used as a fibroblast model. We found that the PDT also induces toxicity in NIH3T3 cells (Fig. 5a, b), but NIH3T3 cells seem to be more sensitive to PDT than TG/HA-VSMCs. Even $17 \mu \mathrm{M}$ chlorin e6 in combination with a $2-\mathrm{J} / \mathrm{cm}^{2}$ light dose induced phototoxicity of $80 \%$ of NIH3T3 cells (Fig. 5a). Also, the conditions which were optimal for the PDT of TG/HA-VSMCs $(170 \mu \mathrm{M}$ of 

apoptosis-related events after PDT $(170 \mu \mathrm{M}$ chlorin e6 and $2-\mathrm{J} / \mathrm{cm}^{2}$ light dose of TG/HAVSMCs: a phosphatidylserine externalization and membrane integrity assessed by staining with Annexin V-FITC conjugate $(\mathrm{FL}-1 \mathrm{H}$, green fluorescence) and propidium iodide staining (FL-3H, far red fluorescence). b Mitochondrial potential using an IC-1 probe (FL-2H, red fluorescence). c Caspase-3 activity (dark bar), the light gray bar represents caspase-3-like activity observed in the presence of the caspase- 3 inhibitor Ac-DEVD-CHO. Averages from representative experiments are shown. d DNA fragmentation observed $18 \mathrm{~h}$ after PDT by propidium iodide (FL2H, red fluorescence)
Fig. 4 Assessment of
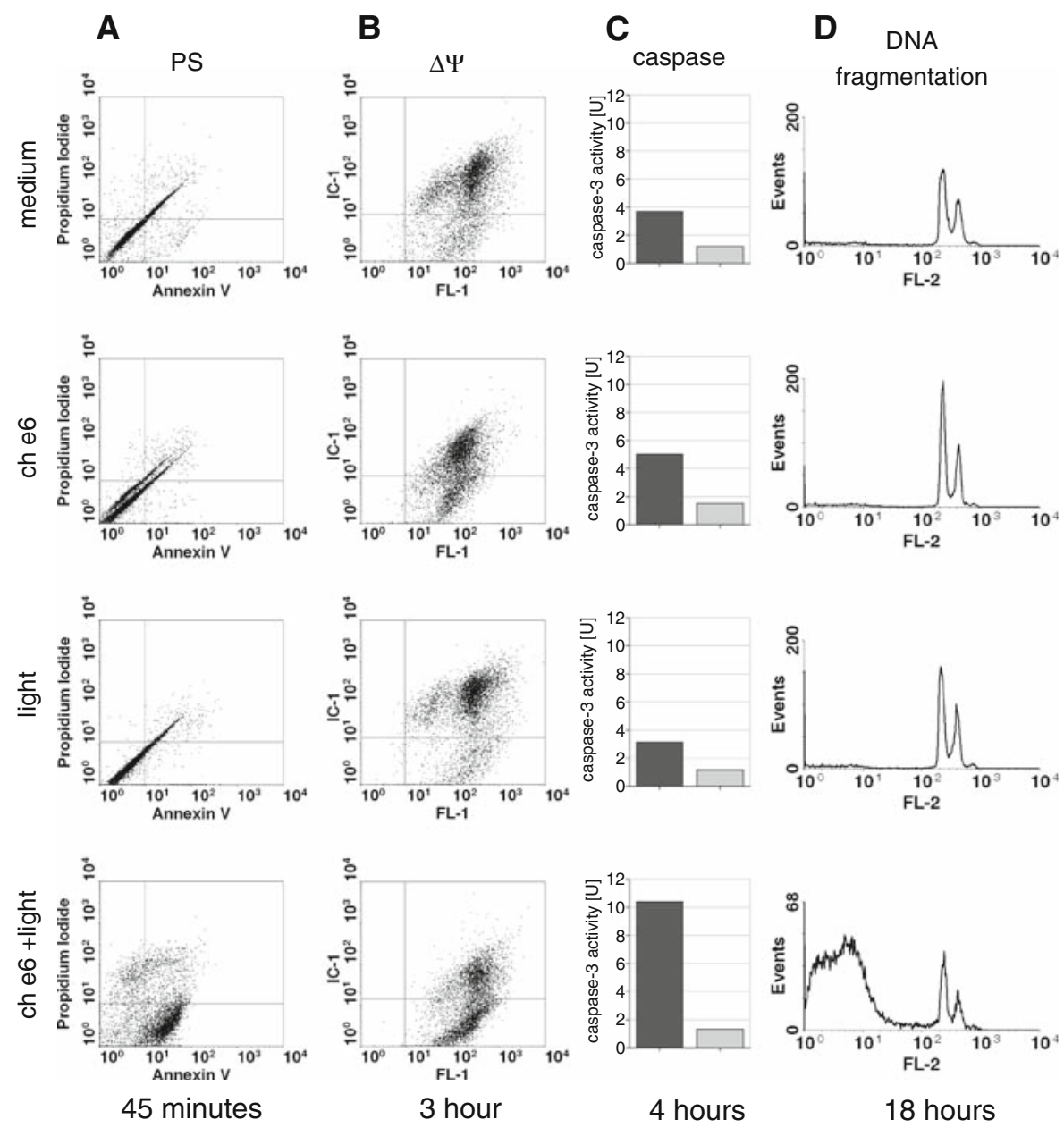

45 minutes

chlorin e6 and a light dose of $1.1 \mathrm{~J} / \mathrm{cm}^{2}$ ) had toxic effect on $80 \%$ of the NIH3T3 cells (Fig. 5b). These findings were reflected by the results of Annexin V/PI staining, in which increases in both Annexin V-positive and Annexin V-PI double-positive cells were observed (Fig. 5c). It is worth mentioning that the observed percentages of both Annexin V-positive populations were larger than those for TG/HAVSMC. We did not observe phosphatidylserine presentation in any of the three control samples (data not shown). However, illumination of photosensitized cells generated much less (only a twofold increase) ROS than in TG/HAVSMCs. There was also a significant increase in detectable ROS in non-illuminated but photosensitized cells, suggesting that, in contrast to TG/HA-VSMCs, the presence of photosensitizer alone may have impact on the condition of NIH3T3 cells (Fig. 5d). At the same time, regardless of chlorin e6 concentration, we could also not detect caspase- 3 activation in the photosensitized and illuminated NIH3T3 fibroblasts (Fig. 5e); moreover, most of the observed activity (dark bars) was not inhibited by the caspase-3 inhibitor Ac-DEVD-CHO (gray bars), suggesting impaired caspase- 3 activation.

\section{Discussion}

Photodynamic therapy is a well-established and clinically approved treatment of some types of cancer, premalignant conditions, and macular degeneration (Biel 2007; Fien and Oseroff 2007; Taub 2007; Woodburn et al. 1998). In cardiology there are increasing attempts to design a PDT for the treatment of restenosis (Pai et al. 2005; Rockson et al. 2000; Sobeh et al. 1995; Zubilewicz et al. 2001). To date, the preferred option of the treatment of post-PCI restenosis has been the use of drug-eluting stents (DESs) delivering antiproliferative agents to the vessel wall. The introduction of the first generation of DESs, delivering rapamycin or paclitaxel to the vessel wall, to clinical practice significantly reduced the incidence of restenosis. However, complete inhibition of the healing response and delayed endothelial regeneration of the thrombogenic surface of the stent may increase the risk of late thrombosis $(>1$ year after the index PCI) and vessel occlusion (Hill et al. 2007; Schiele 2005; Toutouzas et al. 2004). The problem of lifethreatening in-stent thrombosis has raised concerns about the safety of DESs (Weisz and Stone 2008), in light of 
Fig. 5 NIH3T3 response to chlorin e6 PDT. a Results of MTS assay, illumination (laser, $2 \mathrm{~J} / \mathrm{cm}^{2}$ ) in the presence (triangles) or absence (squares) of the indicated concentration of chlorin e6. b Toxicity induced in the cell population after illumination with the indicated light dose in the presence (triangles) or absence (squares) of $170 \mu \mathrm{M}$ chlorin e6; c Phosphatidylserine presentation and membrane integrity test of NIH3T3 cells subjected to PDT $(170 \mu \mathrm{M}$ chlorin e6 and $2 \mathrm{~J} / \mathrm{cm}^{2}$ ). Percentages of populations are shown. A representative result of one of three independent experiments is shown. d Reactive oxygen species (ROS) as 485-/535-nm fluorescence of $\mathrm{H}_{2}$ DCFDA generated in photosensitized and/or illuminated samples. e Caspase- 3 activity measured in lysates photosensitized and/or illuminated NIH3T3 cells (dark bar). The light gray bar represents caspase-3-like activity observed in the presence of the caspase- 3 inhibitor Ac-DEVD-CHO
A

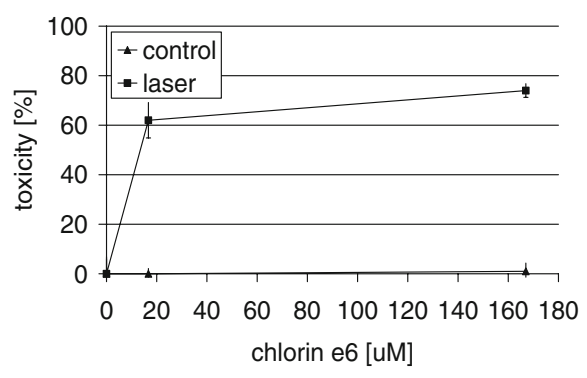

B

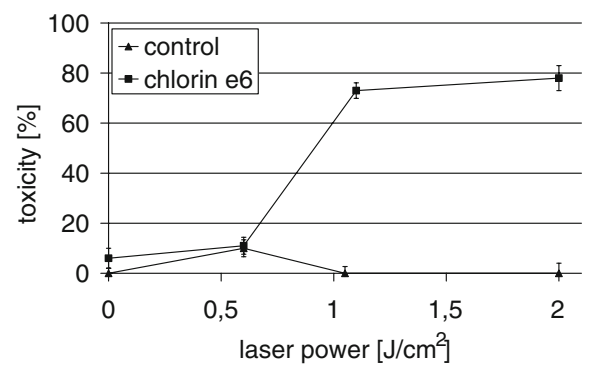

2

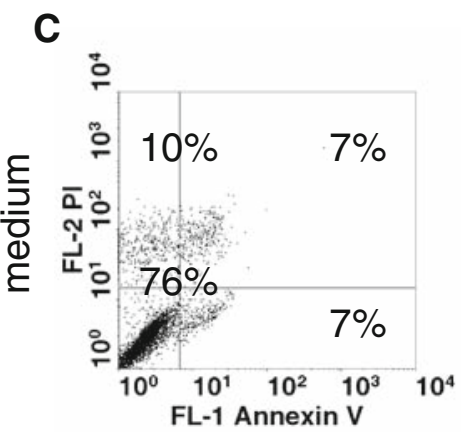

D ROS generation

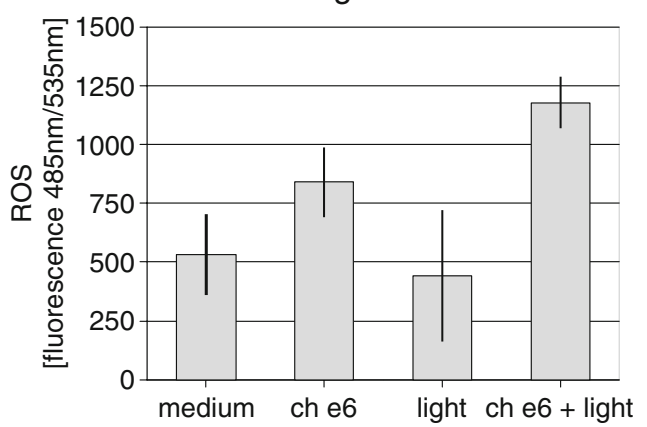

E

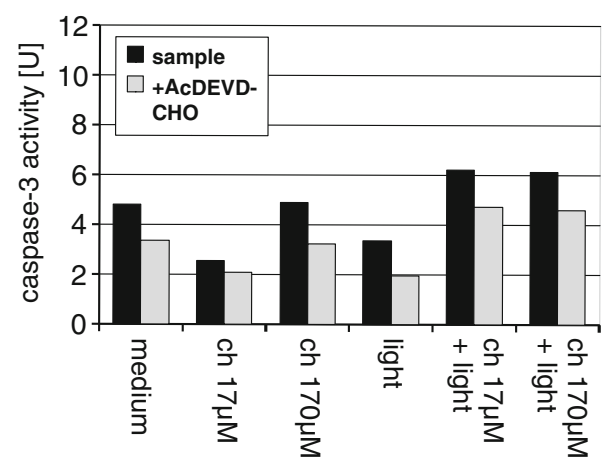

which increased efforts to design an alternative form of treatment of restenosis have become even more urgent.

As the vascular wall's proliferative response to injury was defined against the background of restenosis, selective antiproliferative treatment seems to hold promise to combat this unsolved issue. Here we attempted to test the suitability of chlorin e6 in the PDT of restenosis. Chlorin e6 is a well-known and widely used photosensitizing agent; however, its mode of action on human VSMCs has never been tested. We designed an in vitro experimental model to study the effect of chlorin e6 on human vascular cells, as smooth muscle cells were shown to be the first population responding to vessel injury and to be crucial in the development of restenosis (Landry et al. 1997). It was shown that targeting this population of vascular cells is an effective strategy to prevent restenosis. The first signs of dark toxicity were noted at a concentration of $84 \mu \mathrm{M}$. We found that despite the lack of any carrier, such as liposomes, and despite the presence of serum [which may decrease the bioavaibility of chlorin-based compounds (Bose and Dube 2006)], as little as $10 \mathrm{~min}$ of incubation was enough to observe chlorin e6 inside the cells, which makes it suitable for in vivo sensitizing. Studies performed by Dube et al. (2006) on a similar compound, chlorin p6, showed its quick clearance. It could be expected that chlorin e6, which has a very similar structure, will have similar pharmacokinetics properties, which would make it a good choice for PDT. As was shown in previous studies, chlorin e6 may locate to plasma membrane and/or mitochondria (Kessel and Poretz 2000). Our observed staining pattern rather suggests a cytoplasmic location of chlorin e6 in human smooth muscle cells.

The molecular mechanism of chlorin e6-based PDT remains unclear, and most studies concentrate on tumor cells. ROS or singlet oxygen generation and the induction of endoplasmic reticulum stress and mitochondrial 
dysfunction are the most common events. This may lead to the execution of all kinds of cell-death programs, from classical apoptosis to programmed necrosis or autophagy (Buytaert et al. 2007). We studied many aspects of the illumination-induced programmed cell death of VSMCs and found a rapid induction of ROS, probably close to the mitochondria. The earliest observed cell death-related event, 45 min after illumination of the photosensitized cells, was phosphatidylserine presentation on the surface of the cellular membrane. The second, measured $3 \mathrm{~h}$ after illumination, was the loss of mitochondrial potential. Interestingly, both events correspond to the preferred localization of chlorin e6 in cells. About $4 \mathrm{~h}$ after the illumination of photosensitized cells, an approximately threefold increase in caspase-3 activity was observed. As the final hallmark of apoptosis, DNA fragmentation was observed after $18 \mathrm{~h}$ of incubation, showing that chlorin e6based PDT induces rapid classical apoptosis and indicating its suitability for designing and testing animal models of the treatment of restenosis.

It is known that restenosis is a complex disease also involving other populations of cells, such as macrophages (Farb et al. 2002) and fibroblasts (Bayes-Genis et al. 2002; $\mathrm{Li}$ et al. 2000). It is also not clear if targeting cells other than VSMCs may be beneficial in restenosis therapy. We found that chlorin e6-based PDT applied under conditions effective on VSMCs, despite a low generation of ROS, led to a profound phototoxic effect on NIH3T3 fibroblasts. This may suggest that the phototoxicity of chlorin e6 is mediated only by singlet oxygen. Phosphatidylserine presentation and the gradual increase in phototoxicity observed in 24- an 48-h cultures indicates the occurrence of cell death (not shown). In contrast to VSMCs, PDT of NIH3T3 did not result in caspase-3 activation and DNA fragmentation (not shown). This suggests that in the case of chlorin e6-based PDT of the fibroblasts, another cell-death program is initiated, as was observed in HeLa cells under oxidative stress (Chernyak et al. 2006). This is a good illustration of the possibility of inducing a wide range of cell-death programs, including autophagy (Kessel et al. 2000; Kessel and Reiners 2007), as a response to PDT, and it should be noted that further evaluation of cell death in in vitro and in vivo studies should not depend on one assay (largely MTT or MTS), as that may lead to inaccurate results. The nature of the observed differential response may involve, for example, the pattern of expression of Bcl-2 family proteins, especially $\mathrm{Bax}$ and $\mathrm{Bcl}-2$, whose role in PDT-induced apoptosis was shown by Granville et al. (2001b), although the roles of other family members should not be excluded.

Taken together, using an in vitro PDT model based on human VSMCs we showed that the photosensitizer chlorin e6 may be useful in designing PDT for treating restenosis.
In addition, we showed that PDT under conditions which are optimal and effective on VSMCs induces non-apoptotic cell death of NIH3T3 cells. The fibroblasts appeared to be more sensitive to PDT, indicating a source of potential complications of chlorin e6-based PDT in vivo, which targets multiple cell populations.

Acknowledgments The generous gift of the TG/HA-VSMC cell line from Prof. Danuta Duś and Elżbieta Wojdat is gratefully appreciated. This study was supported by Wroclaw Medical University grant no. 1483, IITD program no. 03/2008, and Ministry of Sciences and Higher Education grant no. N N403 186834.

\section{References}

Bayes-Genis A, Campbell JH, Carlson PJ et al (2002) Macrophages, myofibroblasts and neointimal hyperplasia after coronary artery injury and repair. Atherosclerosis 163:89-98

Biel MA (2007) Photodynamic therapy treatment of early oral and laryngeal cancers. Photochem Photobiol 83:1063-1068

Bose B, Dube A (2006) Interaction of chlorin p6 with bovine serum albumin and photodynamic oxidation of protein. J Photochem Photobiol B 85:49-55

Buytaert E, Dewaele M, Agostinis P (2007) Molecular effectors of multiple cell death pathways initiated by photodynamic therapy. Biochim Biophys Acta 1776:86-107

Chen Z, Woodburn KW, Shi C et al (2001) Photodynamic therapy with motexafin lutetium induces redox-sensitive apoptosis of vascular cells. Arterioscler Thromb Vasc Biol 21:759-764

Chernyak BV, Izyumov DS, Lyamzaev KG et al (2006) Production of reactive oxygen species in mitochondria of HeLa cells under oxidative stress. Biochim Biophys Acta 1757:525-534

Cunderlíková B, Gangeskar L, Moan J (1999) Acid-base properties of chlorin e6: relation to cellular uptake. J Photochem Photobiol B 53:81-90

Derkacz A, Protasiewicz M, Kipshidze N et al (2005) Endoluminal phototherapy for prevention of restenosis: preliminary results at 6-month follow-up. Photomed Laser Surg 23:536-542

Dube A, Sharma S, Gupta PK (2006) Evaluation of chlorin p6 for photodynamic treatment of squamous cell carcinoma in the hamster cheek pouch model. Oral Oncol 42:77-82

Eton D, Shim V, Maibenco TA et al (1996) Cytotoxic effect of photodynamic therapy with Photofrin II on intimal hyperplasia. Ann Vasc Surg 10:273-282

Farb A, Weber DK, Kolodgie FD et al (2002) Morphological predictors of restenosis after coronary stenting in humans. Circulation 105:2974-2980

Fien SM, Oseroff AR (2007) Photodynamic therapy for nonmelanoma skin cancer. J Natl Compr Canc Netw 5:531-540

Granville DJ, Cassidy BA, Ruehlmann DO et al (2001a) Mitochondrial release of apoptosis-inducing factor and cytochrome $\mathrm{c}$ during smooth muscle cell apoptosis. Am J Pathol 159:305-311

Granville DJ, Ruehlmann DO, Choy JC et al (2001b) Bcl-2 increases emptying of endoplasmic reticulum $\mathrm{Ca} 2+$ stores during photodynamic therapy-induced apoptosis. Cell Calcium 30:343-350

Gurinovich GP, Zorina TE, Melnov SB et al (1992) Photodynamic activity of chlorin e6 and chlorin e6 ethylenediamide in vitro and in vivo. J Photochem Photobiol B 13:51-57

Heckenkamp J, Leszczynski D, Schiereck J et al (1999) Different effects of photodynamic therapy and gamma-irradiation on vascular smooth muscle cells and matrix: implications for inhibiting restenosis. Arterioscler Thromb Vasc Biol 19:2154-2161 
Heckenkamp J, Aleksic M, Gawenda M et al (2004) Modulation of human adventitial fibroblast function by photodynamic therapy of collagen matrix. Eur J Vasc Endovasc Surg 28:651-699

Hill RA, Boland A, Dickson R et al (2007) Drug-eluting stents: a systematic review and economic evaluation. Health Technol Assess 11:xi-221

Kereiakes DJ, Szyniszewski AM, Wahr D et al (2003) Phase I drug and light dose-escalation trial of motexafin lutetium and far red light activation (phototherapy) in subjects with coronary artery disease undergoing percutaneous coronary intervention and stent deployment: procedural and long-term results. Circulation 108:1310-1315

Kessel D, Poretz RD (2000) Sites of photodamage induced by photodynamic therapy with a chlorin e6 triacetoxymethyl ester (CAME). Photochem Photobiol 71:94-96

Kessel D, Reiners JJ (2007) Apoptosis and autophagy after mitochondrial or endoplasmic reticulum photodamage. Photochem Photobiol 83:1024-1028

Kessel D, Woodburn K, Gomer CJ et al (1995) Photosensitization with derivatives of chlorin p6. J Photochem Photobiol B 28:13-18

Kessel D, Luo Y, Mathieu P et al (2000) Determinants of the apoptotic response to lysosomal photodamage. Photochem Photobiol 71:196-200

LaMuraglia GM, Schiereck J, Heckenkamp J et al (2000) Photodynamic therapy induces apoptosis in intimal hyperplastic arteries. Am J Pathol 157:867-875

Landry DB, Couper LL, Bryant SR et al (1997) Activation of the NF-kappa B and I kappa B system in smooth muscle cells after rat arterial injury: induction of vascular cell adhesion molecule-1 and monocyte chemoattractant protein-1. Am J Pathol 151:1085-1095

Li G, Chen SJ, Oparil S et al (2000) Direct in vivo evidence demonstrating neointimal migration of adventitial fibroblasts after balloon injury of rat carotid arteries. Circulation 101:1362-1365

Magaraggia M, Visonà A, Furlan A et al (2006) Inactivation of vascular smooth muscle cells photosensitised by liposomedelivered $\mathrm{Zn}(\mathrm{II})$-phthalocyanine. J Photochem Photobiol B $82: 53-58$
Mansfield R, Bown S, McEwan J (2001) Photodynamic therapy: shedding light on restenosis. Heart 86:612-618

Moussavian M, Casterella P, Teirstein P (2001) Restenosis after angioplasty. Curr Treat Options Cardiovasc Med 3:103-113

Nagae T, Aizawa K, Uchimura N et al (2001) Endovascular photodynamic therapy using mono-L-aspartyl-chlorin e6 to inhibit Intimal hyperplasia in balloon-injured rabbit arteries. Lasers Surg Med 28:381-388

Pai M, Jamal W, Mosse A et al (2005) Inhibition of in-stent restenosis in rabbit iliac arteries with photodynamic therapy. Eur J Vasc Endovasc Surg 30:573-581

Rockson SG, Lorenz DP, Cheong WF et al (2000) Photoangioplasty: an emerging clinical cardiovascular role for photodynamic therapy. Circulation 102:591-596

Schiele TM (2005) Current understanding of coronary in-stent restenosis. Pathophysiology, clinical presentation, diagnostic work-up, and management. Z Kardiol 94:772-790

Sobeh MS, Chan P, Ham RJ et al (1995) Photodynamic therapy in a cell culture model of human intimal hyperplasia. Eur J Vasc Endovasc Surg 9:463-468

Taub AF (2007) Photodynamic therapy: other uses. Dermatol Clin 25:101-109

Toutouzas K, Colombo A, Stefanadis C (2004) Inflammation and restenosis after percutaneous coronary interventions. Eur Heart J 25:1679-1687

Wakamatsu T, Saito T, Hayashi J et al (2005) Long-term inhibition of intimal hyperplasia using vascular photodynamic therapy in balloon-injured carotid arteries. Med Mol Morphol 38:225-232

Weisz G, Stone GW (2008) Safety and efficacy of drug-eluting stents: on-label and off-label perspectives. Rev Cardiovasc Med 9:46-61

Woodburn KW, Fan Q, Kessel D et al (1998) Photodynamic therapy of B16F10 murine melanoma with lutetium texaphyrin. J Invest Dermatol 110:746-751

Zubilewicz T, Wronski J, Bourriez A et al (2001) Injury in vascular surgery-the intimal hyperplastic response. Med Sci Monit $7: 316-324$ 